\title{
Evaluación de la técnica quirúrgica "curetaje" axilar para el tratamiento de la hiperhidrosis axilar, fase retrospectiva
}

Evaluation of a surgical technique for the treatment of axillary hyperhidrosis, retrospective phase

\section{Claudia Andrea Hernández ${ }^{1}$, Luz Marina Gómez², Ángela Londoño³, Natalia Mendoza ${ }^{4}$}

1. Médica dermatóloga, Facultad de Medicina, Escuela de Ciencias de la Salud, Universidad Pontificia Bolivariana, Medellín, Colombia.

2. Médica dermatóloga; profesora titular; jefe, Servicio de Dermatología, Facultad de Medicina, Escuela de Ciencias de la Salud, Universidad Pontificia Bolivariana, Medellín, Colombia.

3. Médica dermatóloga y epidemióloga; docente, Facultad de Medicina, Escuela de Ciencias de la Salud, Universidad Pontificia Bolivariana, Medellín, Colombia.

4. Médica, residente de III año de Dermatología, Facultad de Medicina, Escuela de Ciencias de la Salud, Universidad Pontificia Bolivariana, Medellín, Colombia.

\section{Resumen}

INTRoDUCCIón. La hiperhidrosis axilar es una condición relativamente frecuente y de difícil manejo, que afecta en forma negativa la calidad de vida del paciente y origina estigmas sociales ya que interfiere con las actividades de la vida diaria. El manejo tradicional de la hiperhidrosis axilar se basa en el uso de preparados tópicos. Como terapias de segunda línea se encuentra la toxina botulínica y diversas técnicas quirúrgicas de destrucción local de las glándulas sudoríparas y la simpatectomía torácica. La técnica quirúrgica de "curetaje" (curetagge) axilar, utilizada en el Servicio de Dermatología de la Universidad Pontificia Bolivariana se propone como una opción para aquellos pacientes que no responden al manejo conservador.

OBJETivo. Evaluar una técnica quirúrgica llevada a cabo en la Clínica Universitaria Bolivariana y en la Clínica SOMA para el tratamiento de la hiperhidrosis axilar.

Metodología. Se trata de un estudio descriptivo, realizado en dos fases: retrospectiva y prospectiva con seguimiento al mes y al año. En este manuscrito se presentan los resultados retrospectivos.

RESUltados. Se incluyeron 9 pacientes en la fase retrospectiva del estudio, con 18 unidades quirúrgicas. Todos los pacientes habían recibido algún tipo de tratamiento previo. La seriedad de la enfermedad antes de la cirugía era grave en todos los pacientes; al mes de la cirugía era leve en cuatro pacientes y moderada en 5; al año de la cirugía, era leve en dos pacientes y moderada en siete. Siete pacientes consideraron leve el dolor posquirúrgico y hubo una baja tasa de complicaciones.

Conclusión. La técnica quirúrgica propuesta para el manejo de la hiperhidrosis axilar es efectiva, con bajo porcentaje de complicaciones, la cual puede ser recomendada como segunda línea de tratamiento para pacientes que no respondan al manejo conservador.

PALABRAS CLAVE: hiperhidrosis axilar; tratamiento; cirugía.

\section{Summary}

OBJETIVE: To evaluate a surgical technique applied at the Clínica Universitaria

\section{Correspondencia:}

Claudia Andrea Hernández

Email:

mpcah@hotmail.com

Recibido: 28 de junio de 2012.

Aceptado: 8 de agosto de 2012.

No se reportan conflictos de intereses. 
Bolivariana and Clínica SOMA for the treatment of axillary hyperhidrosis.

METHOdology: This is a two phase descriptive study: retrospective and prospective, with a month and one year follow-up.

RESULTS: Nine patients were admitted in the retrospective phase of the study, for a total of 18 surgical units. All patients had received previous treatment. The severity of the disease before surgery was high in all cases; one month after surgery, the severity was mild in four patientes and moderate in five; one year after surgery, the severity was mild in two patients and moderate in seven. Seven patients considered postoperative pain was mild and there was a low complication rate.

CoNCLUSION: The proposed surgical technique for the management of axillary hyperhidrosis, is effective, has few complications and can be recommended as a second line therapy for patients that do not respond to conservative therapies.

KEY WORDS: Axillary hyperhidrosis, treatment, surgery.

\section{Introducción}

La hiperhidrosis primaria (exceso de sudoración de causa desconocida) es una condición relativamente frecuente que puede presentarse hasta en el $3 \%$ de la población ${ }^{1}$. Se presenta de manera focal, principalmente en axilas (50-80 \%), palmas y plantas, aunque también puede presentarse en otras áreas corporales como la cara ${ }^{2-5}$.

Se considera una anormalidad funcional, no inflamatoria de las glándulas sudoríparas. La causa subyacente no es clara y se cree que se debe a un estímulo simpático extremo de falta de regulación térmica o a una disfunción compleja del sistema nervioso periférico. Además, como el trastorno no se presenta durante el sueño, se ha sugerido que las emociones pueden jugar un papel importante en la patogénesis. A nivel cerebral, la corteza anterior del cíngulo, la cual, a su vez, es regulada por el hipotálamo anterior parece ser la responsable de este vínculo $^{2-4}$. De 60 a $80 \%$ de los pacientes tienen historia familiar de hiperhidrosis, lo que sugiere una herencia autosómica dominante con penetrancia incompleta ${ }^{6}$.

Hund, et al., definen la hiperhidrosis axilar como una tasa de sudoración mayor de 50-100 $\mathrm{ml}$ en 5 minutos por axila, medido por estudios de gravimetría ${ }^{7}$. No tiene predilección por sexo ni raza y típicamente se inicia durante la pubertad o después de ella. La parte central de la axila es responsable de 70 a $80 \%$ de la secreción del sudor ya que posee una concentración glandular mucho mayor que la periferia ${ }^{8}$. Generalmente, la axila derecha produce más sudor que la izquierda y en casos raros una axila puede ser hiperhidrótica y la otra hipohidrótica o anhidrótica9.

En todos los casos esta condición afecta la calidad de vida a nivel social y laboral, originando problemas psi- cosociales puesto que se asocia con humedad permanente, sensación de gotas de sudor deslizándose sobre la superficie cutánea, daño de las vestimentas por decoloración o por tinción amarillo-café (cromhidrosis) y bromhidrosis (mal olor) debida a la presencia de colonización bacteriana ${ }^{2-5}$.

Los criterios diagnósticos de hiperhidrosis primaria localizada son $^{10}$ :

- Sudoración excesiva visible y focal.

- Al menos, 6 meses de duración.

- Sin causa secundaria aparente.

Asociada a, por lo menos, dos de los siguientes criterios:

- Sudoración bilateral y simétrica.

- Ausencia de sudoración nocturna.

- Al menos, un episodio semanal.

- Inicio a una edad menor o igual a 25 años.

- Historia familiar positiva.

- Deterioro de las actividades de la vida diaria.

El tratamiento de la hiperhidrosis es difícil. Como primera línea se utilizan medicamentos tópicos: antitranspirantes, sales de aluminio y astringentes, los cuales tienen como ventaja un bajo costo y fácil acceso pero su uso se limita por una corta duración de acción (aproximadamente, 1 semana) y baja efectividad, además de efectos secundarios como irritación, sensación de quemazón o disestesias en la gran mayoría de los $\operatorname{casos}^{11-12}$. La toxina botulínica se considera de segunda línea y, aunque es más efectiva que los tópicos, es de alto costo y de poca duración ${ }^{13-15}$.

La cirugía se considera una alternativa terapéutica de 


\begin{tabular}{|c|c|}
\hline Grados & \\
\hline 1: leve & La sudoración nunca es notoria y nunca interfiere con las actividades de la vida diaria. \\
\hline 2: moderado & La sudoración es tolerable e interfiere ocasionalmente con las actividades de la vida diaria. \\
\hline 3: grave & La sudoración es poco tolerable e interfiere frecuentemente con las actividades de la vida diaria. \\
\hline 4: grave & La sudoración es intolerable y siempre interfiere con las actividades de la vida diaria. \\
\hline
\end{tabular}

TABLA 1. Escala de gravedad de la hiperhidrosis (Hyperhidrosis Disease Severity Scale, HDSS)

segunda linea en casos resistentes al tratamiento. En esta modalidad se encuentra:

- La simpatectomía transtorácica: es la última opción ya que se asocia con complicaciones sistémicas tales como neumotórax, síndrome de Horner, hiperhidrosis compensatoria y recurrencias ${ }^{16,17}$

- Las técnicas locales para la remoción de las glándulas sudoríparas (escisión en bloque de la piel afectada, o "curetaje” o liposucción axilar): son consideradas el método quirúrgico de elección ${ }^{18,19}$.

La técnica quirúrgica de "curetaje" axilar de las glándulas sudoríparas, propuesta inicialmente por Jemec en 1975 consiste en dos incisiones de $1,5 \mathrm{~cm}$ en los extremos proximal y distal de la axila bajo anestesia local o general, y, posteriormente, el "curetaje" del tejido subcutáneo ${ }^{20}$. Dicha técnica fue modificada en 1991 por L. M. Gómez y F. Gómez, cambiando el número de incisiones a una, orientada sobre los pliegues anatómicos ${ }^{8,9}$; fue implementada en el Servicio de Dermatología de la Clínica Universitaria Bolivariana y en la Clínica SOMA.

El objetivo de esta investigación es evaluar los resultados obtenidos en los pacientes con diagnóstico de hiperhidrosis axilar que no responden al manejo conservador, atendidos entre junio de 2008 y enero de 2013 en estas dos instituciones.

\section{Métodos}

Se trata de un estudio observacional descriptivo, llevado a cabo en dos fases:

- Retrospectiva: entre junio de 2008 y enero de 2011, cuyos resultados son los que se presentan en este artículo.

- Prospectiva: entre febrero de 2011 y enero de 2013, cuyos resultados se entregarán en mayo de 2013.

Los criterios de inclusión del estudio fueron los siguientes:

- Pacientes de la Clínica Universitaria Bolivariana y la Clínica Soma con diagnóstico de hiperhidrosis axilar operados entre junio de 2008 y enero de 2011 con la misma técnica quirúrgica (fase retrospectiva).
- Pacientes remitidos a la Clínica Universitaria Bolivariana y Clínica Soma con diagnóstico de hiperhidrosis axilar por otro dermatólogo con orden para el procedimiento y que fueran candidatos a tratamiento luego de una evaluación clínica, y que posteriormente hayan sido operados entre junio de 2008 y enero de 2011 (fase retrospectiva).

- Pacientes con indicación de manejo quirúrgico que deseen voluntariamente realizárselo y participar en la investigación.

- Pacientes con hiperhidrosis axilar que no respondan a manejos tópicos de primera línea o que no sean candidatos al uso de toxina botulínica de tipo $\mathrm{A}$.

Los criterios de exclusión del estudio fueron:

- Pacientes alérgicos al yodo en quienes está contraindicada la aplicación del test de Minor.

- Pacientes con hiperhidrosis generalizada (incluyendo axilar).

- Pacientes con hiperhidrosis secundaria a enfermedades de base.

- Pacientes con hiperhidrosis axilar que hayan sido manejados previamente con "curetaje" o liposucción axilar.

De todos los pacientes de esta fase se obtuvieron los siguientes datos: sexo, edad, tiempo de evolución de la enfermedad y tratamientos recibidos antes del procedimiento quirúrgico; se les aplicó una escala validada de gravedad de la hiperhidrosis (Hyperhidrosis Disease Severity Scale, HDSS) ${ }^{21}$. Esta escala ha sido utilizada en múltiples estudios epidemiológicos de la hiperhidrosis y se calcula que hasta 55,2 \% de los pacientes con hiperhidrosis tienen un puntaje de 4 (grave) ${ }^{5,22}$.

Además, se les tomaron fotografías del resultado del test de Minor (prueba de yodo) antes de la cirugía y después de ella. Esta prueba consiste en la aplicación de solución de lugol en la región axilar, previa limpieza y secado del área. Se deja secar, se esparce el almidón y se espera a que el paciente sude. El almidón y el yodo interactúan en presencia de sudor generando una reacción que tiñe de color azul violeta el área de piel con mayor sudoración, facilitando la detección de la zona específica por tratar ${ }^{8,9}$. 

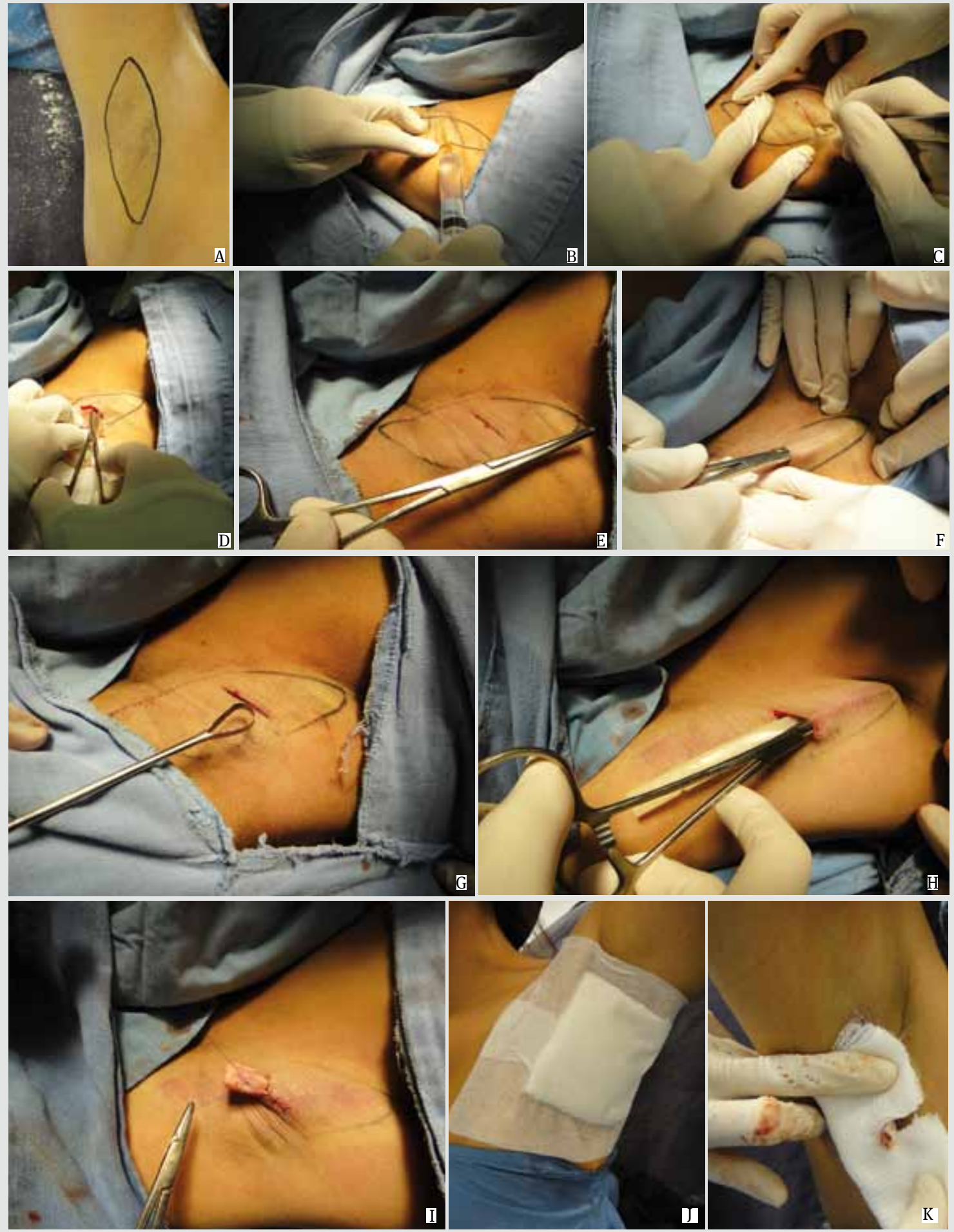

FIGURA 1. Pasos del procedimiento quirúrgico utilizado en el estudio "Evaluación de la técnica quirúrgica curetaje axilar usada para hiperhidrosis axilar en pacientes atendidos en la Clínica Universitaria Bolivariana y Clínica SOMA de Junio de 2008 a Enero de 2013”. A. Se coloca el paciente en posición supina con los brazos en abducción de $90^{\circ}$. Se limpia y seca la región axilar.Se practica el test de Minor o de yodo y almidón.Se delinea la zona de máxima sudoración con un marcador de piel resistente al agua.Asepsia del área. B. Se aplica anestesia local (lidocaína más bupivacaína con epinefrina). C. Se hace una incisión transversal de 2 a $3 \mathrm{~cm}$ en la parte central de la axila, siguiendo los pliegues axilares. D,E,F. Se hace divulsión con tijera curva y con pinza de Rochester, en la unión de la dermis con el tejido celular subcutáneo, al área demarcada con el test de Minor. G. Se practica "curetaje" con legra o "cureta" (curette) de Sim, de la cara dérmica. Se debe evitar hacerlo hacia la cara hipodérmica para no causar daños al paquete vascular y nervioso de la axila. H. Se coloca el dren de Penrose fijado a la piel. Este se retira una vez haya disminuido la secreción, entre 48 a 72 horas, aproximadamente. I,J,K. Curación con gasas compresivas. Tratamiento con antibióticos de amplio espectro por 5 a 7 días, analgésicos, anti inflamatorios y medidas locales. 
Los pacientes se obtuvieron de la base de datos de las salas de cirugía donde se realizó el procedimiento, que cumplían los criterios de inclusión y de exclusión. Se revisaron las historias clínicas, obteniendo algunos datos y, además, se les contactó telefónicamente, preguntándoles por la presencia de dolor, infección, hematomas, seromas, dehiscencia de la herida quirúrgica, presencia de ampollas, aspecto de las cicatrices (aumentada de tamaño, engrosada o levantada), alteraciones de la pigmentación axilar (hipopigmentación e hiperpigmentación), trastornos de la sensibilidad (disminución de la sensación) y disminución del vello axilar luego del procedimiento.

La satisfacción obtenida se evaluó preguntando por la conformidad con los resultados estéticos y funcionales según la escala validada de gravedad de la hiperhidrosis (HDSS) ${ }^{21}$ (TABLA 1) y los resultados comparativos de las fotografías prequirúrgicas y posquirúrgicas del test de Minor.

\section{Técnica quirúrgica}

La técnica quirúrgica utilizada en el presente estudio consiste en la remoción de tejido celular subcutáneo axilar por "curetaje" rúrgico se muestran en la FIGURA 1.

\section{Análisis}

Cada área intervenida, axila derecha o izquierda, fue considerada como una "unidad quirúrgica" para la evaluación de los resultados. Todas las variables fueron evaluadas por los investigadores, buscando la presencia o ausencia de cada una de ellas. Se definió el resultado final según la escala de gravedad y la respuesta en el test de Minor. El análisis de los datos se efectuó con el programa SPSS ${ }^{\mathrm{TM}}$, versión 18.

Para las variables cuantitativas, edad y tiempo de evolución, se obtuvo el promedio. Las variables cualitativas como dolor, infección, hematomas, seromas, dehiscencia de la herida quirúrgica, presencia de ampollas, aspecto de las cicatrices -aumento de tamaño, de grosor o sobrelevantada-, alteraciones de la pigmentación axilar -hipopigmentación e hiperpigmentación-, trastornos de la sensibilidad -disminución de la sensación-, disminución del vello axilar luego del procedimiento, se midieron según el porcentaje de presentación.

La respuesta a la cirugía se midió según la diferencia en el puntaje de la escala de gravedad antes de la cirugía y después de ella por medio de la comparación de fotografías del test de Minor antes del procedimiento y después del mismo.

\section{Aspectos éticos}

El trabajo cumple con los principios éticos enunciados en el Informe Belmont del 18 de abril de 1979, de la Declaración de Helsinki y de la Resolución oo8430 de 1993, por la cual el Ministerio de Salud de la República de Colombia establece las normas científicas, técnicas y administrativas para la investigación en salud.

Es considerada una investigación de riesgo mínimo: en $\mathrm{su}$ fase retrospectiva emplea el registro de datos mediante un procedimiento que se considera de rutina para el manejo de esta entidad. Fue aprobado por el Comité de Ética de la Escuela de Ciencias de la Salud de la Universidad Pontificia Bolivariana.

\section{Resultados}

Se incluyeron 9 pacientes en la fase retrospectiva del estudio, para un total de 18 unidades quirúrgicas. Seis pacientes eran mujeres y tres hombres; el rango de edad fue de 15 a 54 años, cuatro se encontraban entre los 21 y los 30 años. El tiempo de evolución de la enfermedad fue de 19 años, en promedio (rango, 2 a 40 años). Cuatro pacientes presentaban un tiempo de evolución menor de 5 años y sólo uno un tiempo de evolución mayor de 20 años. En todos los casos la enfermedad inició en la adolescencia.

Doce de las 18 unidades quirúrgicas presentaba bromhidrosis asociada a la hiperhidrosis, y 16 de las 18 presentaba cromhidrosis. Todos los pacientes habían recibido algún tipo de tratamiento previo, ocho con sales de aluminio tópicas, uno con combinación de sales con clindamicina tópica, dos con propanolol oral y uno con toxina botulínica de tipo A. En cuanto al dolor postquirúrgico, siete pacientes lo consideraron leve, uno moderado y uno intenso.

En cuanto a la escala de gravedad de la hiperhidrosis antes de la cirugía, todos los pacientes manifestaron una enfermedad grave (ocho con puntaje de $4 \mathrm{y}$ uno con puntaje de 3). Al mes de la cirugía todos los pacientes refirieron mejoría, cuatro manifestaron una gravedad leve y cinco moderada. Al año de la cirugía, dos pacientes manifestaron una gravedad leve y siete, moderada.

En cuanto a las complicaciones, estas se presentaron en 8 de las 18 unidades intervenidas; en 6 de 18 se presentaron ampollas asociadas a la aplicación del vendaje; en 4 de 18 hubo equimosis en la herida quirúrgica y en varios casos fue concomitante la presencia de estas dos complicaciones. En cuanto a la cicatriz, en 16 de 18 las cicatrices fueron de aspecto normal, en 3 de 18, cicatrices hipertróficas y en 1 de 18 , de tipo queloide; en 9 de 18 hubo hiperpigmentación en el área tratada. Ningún paciente presentó hematomas, dehiscencia de la herida, hipopigmentación, cambios de la sensibilidad, seromas, infección o necrosis (TABLA 2).

De los seis pacientes con bromhidrosis, dos refirieron 
persistencia de esta sintomatología después de la cirugía, mientras que todos los pacientes con cromhidrosis refirieron resolución de este síntoma. Cuatro pacientes manifestaron disminución del vello axilar después de la cirugía.

En el test de Minor se observó una disminución en el área demarcada de sudoración al mes de la cirugía y resultados similares al año de la intervención (FIGURA 2).

\section{Discusión}

La hiperhidrosis axilar es una entidad que causa incapacidades y es de difícil tratamiento. En nuestro trabajo pudimos observar un comportamiento epidemiológico similar a lo descrito en la literatura: una condición que se inicia durante la pubertad, tiene un curso crónico, con mala respuesta a los tratamientos conservadores y que conlleva a una gran morbilidad ya que interfiere con las actividades de la vida diaria. Pudimos observar una asociación frecuente de la hiperhidrosis con cromhidrosis $(88,9 \%)$ o con la bromhidrosis $(66,7 \%)$. Es característica la historia de múltiples consultas y tratamientos, sin una solución definitiva. Todos los pacientes del estudio recibieron manejo conservador antes de la cirugía. Sólo uno de los pacientes recibió manejo con toxina botulínica tipo A, el resto de los casos refirió limitantes económicas para acceder a este tratamiento.

El "curetaje" axilar tiene como ventaja que produce menos cicatrices que la escisión de la piel axilar y que la liposucción, no se asocia con complicaciones sistémicas comparado con la simpatectomía y puede repetirse en caso de ser necesario ya que produce cicatrices mínimas sin contracturas. Comparado con la liposucción, la cicatriz es más estética, hay una mayor satisfacción de los pacientes, menos efectos secundarios y una menor incapacidad $^{18,19}$. La liposucción ha mostrado, contrario al "curetaje", mejores resultados para el manejo de la bromhidrosis ${ }^{23}$. Comparado con la toxina botulínica, el "curetaje" tiene una efectividad similar, pero tiene la ventaja de que los resultados son permanentes en la mayoría de los casos y no temporales como con la toxina ${ }^{13-15}$.

En 1978, Jemec y Holm evaluaron los resultados del "curetaje" axilar en 161 pacientes (322 unidades quirúrgicas), y encontraron que el $36 \%$ no estaba satisfecho con los resultados. Se reportaron eventos adversos en 18 casos, que incluían abscesos y necrosis importante que requirió manejo con injertos ${ }^{24}$.

Por el contrario, nuestros pacientes presentaron

\begin{tabular}{ll}
\hline & $\mathrm{n}$ \\
\hline Hiperpigmentación & 9 \\
Ampollas & 6 \\
Cicatriz hipertrófica & 3 \\
Cicatriz queloide & 1 \\
Equimosis & 4 \\
Hematomas & 0 \\
Dehiscencia & 0 \\
Infección & 0 \\
Seromas & 0 \\
Disestesia & 0 \\
Necrosis & 0 \\
Hipopigmentación & 0 \\
\hline \hline
\end{tabular}

TABLA 2. Complicaciones de la cirugía por unidad quirúrgica.

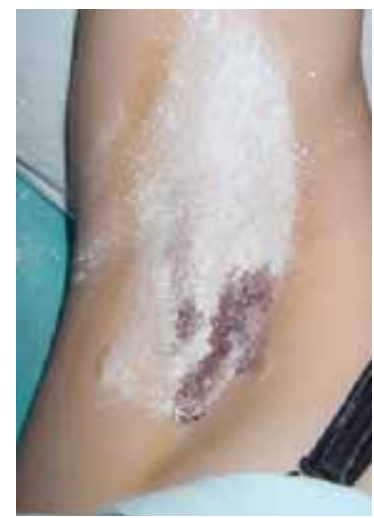

ANTES DE LA CIRUGÍA

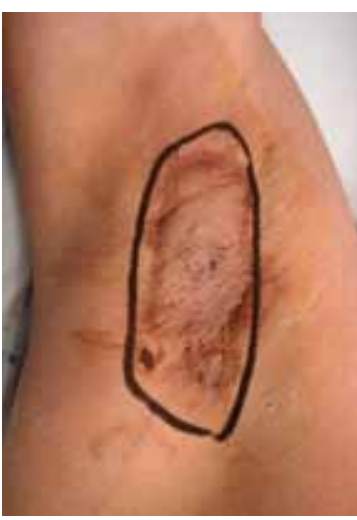

ANTES DE LA CIRUGÍA

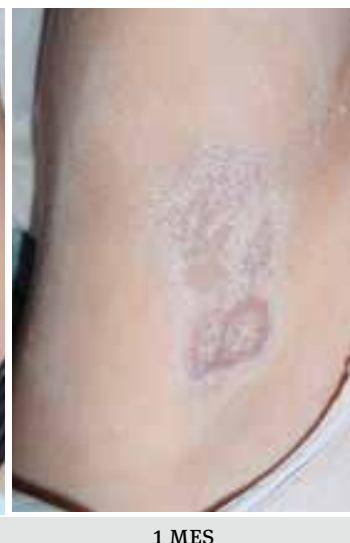

1 MES

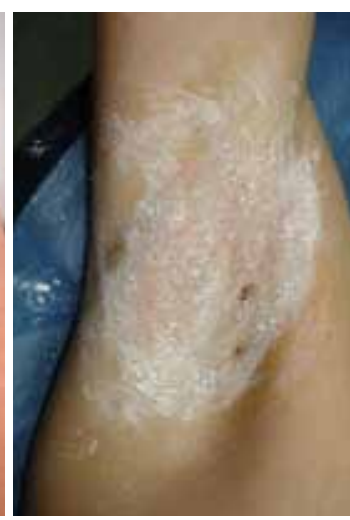

1 AÑo

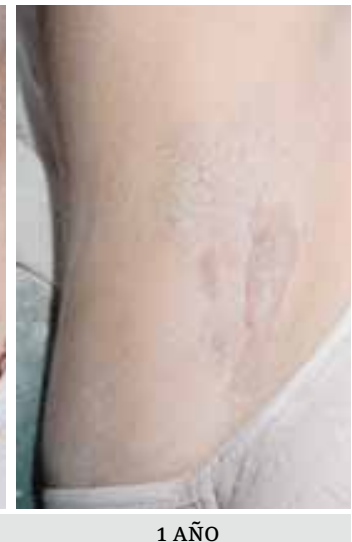

FIGURA 2. Resultados del test de Minor antes y después de la cirugía. 


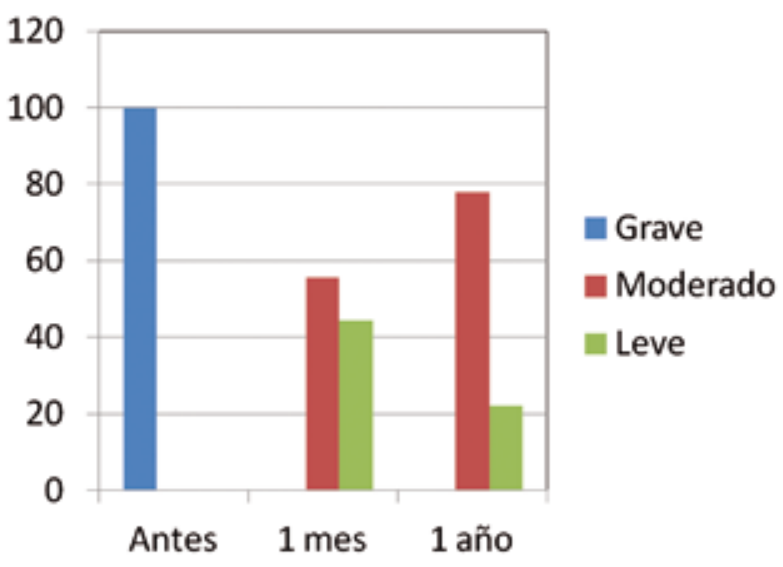

FIGURA 3. Seriedad de la hiperhidrosis medida en porcentajes antes de la cirugía, al mes y al año de la intervención.
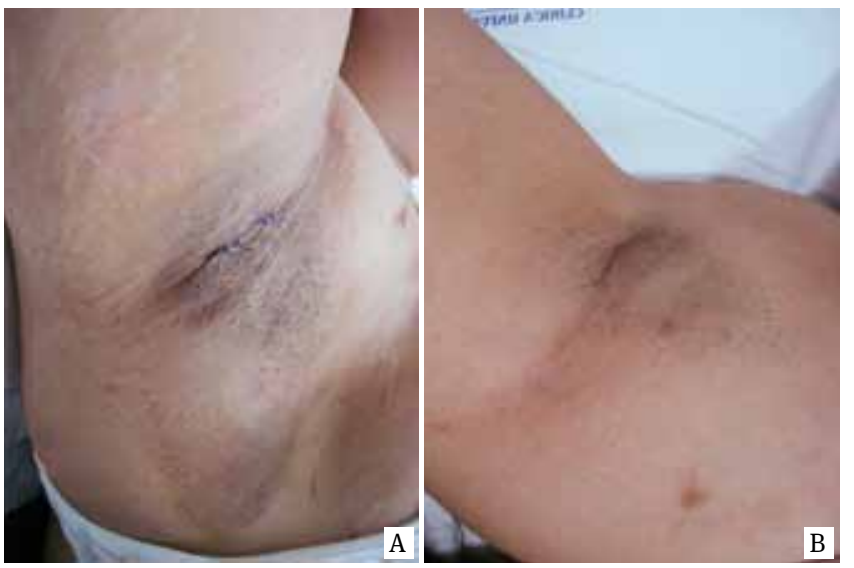

FigurA 4. Aspecto de la herida quirúrgica a los pocos días (A) y al mes de la cirugía (B) en un mismo paciente.

buena evolución posoperatoria, con buena tolerancia al dolor y pocos efectos secundarios. La complicación más frecuente fue la hiperpigmentación en el área tratada, seguida de la presencia de ampollas y equimosis, y ningún paciente tuvo efectos secundarios significativos. $\mathrm{Al}$ igual que en la literatura, los resultados se mantuvieron al año de seguimiento ${ }^{25}$. A pesar de los buenos resultados obtenidos, debemos tener en cuenta una limitante de este estudio que fue la poca cantidad de unidades quirúrgicas evaluadas.

La máxima disminución de la sudoración fue al mes de la intervención quirúrgica y aunque esta mejoría declinó al año, la seriedad de la enfermedad nunca llegó a ser igual o peor a la que se presentaba antes de la cirugía (FIGURA 3).

Aunque la cirugía evaluada, no se considera un procedimiento de bajo costo por requerir quirófano, a largo
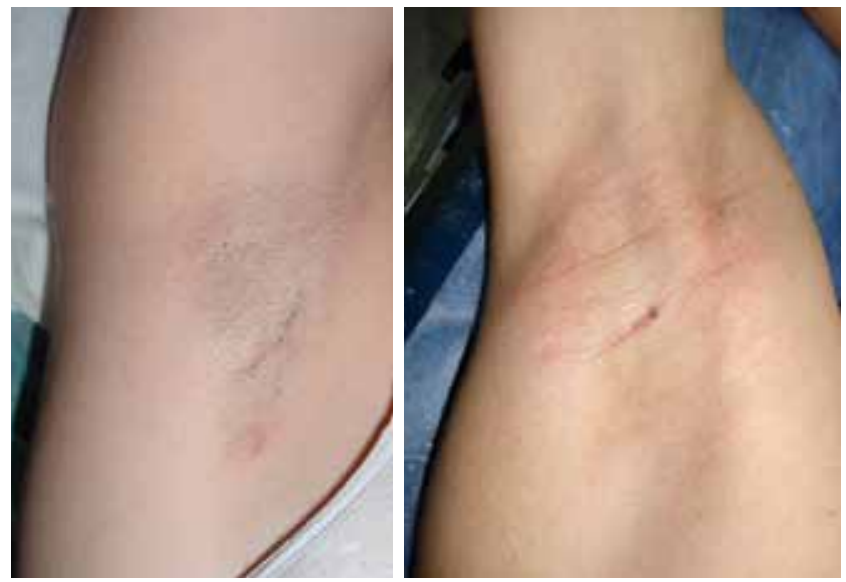

FIGURA 5. Aspecto de las cicatrices a un año de la cirugía en dos pacientes diferentes.

plazo demostró seguridad con pocas complicaciones y resultados sostenidos a un año de la intervención.

En conclusión, la variante de la técnica quirúrgica de Jemec, propuesta en este estudio, demostró ser un procedimiento mínimamente invasor, seguro, eficaz a largo plazo, con una alta satisfacción de los pacientes por los resultados funcionales y estéticos (FIGURAS 4 Y 5), lo que hace de este procedimiento, un excelente método terapéutico para el manejo de la hiperhidrosis axilar en pacientes con enfermedad resistente a los tratamientos tópicos y en pacientes que no sean candidatos a manejo con toxina botulínica de tipo A, y como una opción antes de recurrir a manejos más agresivos como la simpatectomía.

\section{Referencias}

1. Altman RS, Schwartz RA. Emotionally induced hyperhidrosis. Cutis. 2002;69:336-8.

2. Vorkamp T, Foo FJ, Khan S, Schmitto JD, Wilson P. Hyperhidrosis: Evolving concepts and a comprehensive review. Surgeon. 2010;8:287-92.

3. Schlereth T, Dieterich M, Birklein F. Hyperhidrosis-causes and treatment of enhanced sweating. Dtsch Arztebl Int. 2009;106:32-7.

4. Togel B, Greve B, Raulin C. Current therapeutic strategies for hyperhidrosis: A review. Eur J Dermatol. 2002;12:219-23.

5. Strutton DR, Kowalski W, Glaswer DA, Stang PE: US prevalence of hyperhidrosis and impact on individuals with axillary hyperhidrosis: Results from a national survey. J Am Acad Dermatol. 2004; 51:241-8.

6. Hurley HL, Shelley WB. Axillary hyperhidrosis clinical features and local surgical management. Br J Dermatol. 1966;78:127-41.

7. Hund M, Kinkelin I, Naumann M, Hamm H. Definition of axillary hyperhidrosis by gravimetric assessment. Arch Dermatol. 2002;138:539-41.

8. Gómez F, Gómez LM. Hiperhidrosis axilar: un nuevo método de 
tratamiento y revisión de la literatura. Revista Asociación Colombiana de Dermatología. 1991;1:54-7.

9. Toro AM, Gómez LM. Curetaje subcutáneo en el manejo de la hiperhidrosis axilar. Revista Asociación Colombiana de Dermatología. 2007;237-40.

10. Hornberger J, Grimes K, Naumann M, Glaser DA, Lowe NJ, Naver $\mathrm{H}$, et al. Recognition, diagnosis and treatment of primary focal hyperhidrosis. J Am Acad Dermatol. 2004;51:274-86.

11. Boni R. Generalized hyperhidrosis and its systemic treatment. Curr Probl Dermatol. 2002;30:44-7.

12. Flanagan $\mathrm{KH}$, Glaser DA. An open-label trial of the efficacy of $15 \%$ aluminum chloride in $2 \%$ salicylic acid gel base in the treatment of moderate-to-severe primary axillary hyperhidrosis. J Drugs Dermatol. 2009;8:477-80.

13. Naumann MK, Hamm H, Lowe NJ. Effect of botulinum toxin type A on quality of life measures in patients with excessive axillary sweating: A randomized controlled trial. Br J Dermatol. 2002;147:1218-26.

14. Naumann M, Lowe NJ, Kumar CJ, Hamm MD. Botulinum toxin type $A$ is a safe and effective treatment for axillary hyperhidrosis over 16months: A prospective study. Arch Dermatol. 2003;139:731-6.

15. Flanagan $\mathrm{KH}$, King R, Glaser DA. Botulinum toxin type a versus topical $20 \%$ aluminum chloride for the treatment of moderate to severe primary focal axillary hyperhidrosis. J Drugs Dermatol. 2008;7:221-7.

16. Herbst F, Plas EG, Fugger R, Fritsch A. Endoscopic thoracic sympathectomy for primary hyperhidrosis of the upper limbs. A critical analysis and long-term results of 480 operations. Ann Surg. 1994;220:86-90.
17. Dumont P, Denoyer A, Robin P. Long-term results of thoracoscopic sympathectomy for hyperhidrosis. Ann Thorac Surg. 2004;78:1801-7.

18. Swinehart JM. Treatment of axillary hyperhidrosis: combination of the starch-iodine test with the tumescent liposuction technique. Dermatol Surg. 2000;26:392-6.

19. Lee MR, Ryman WJ. Liposuction for axillary hyperhidrosis. Australas J Dermatol. 2005;46:76-9.

20. Jemec B. Abrasio axillae in hyperhidrosis. Scand J Plast Reconstr Surg. 1975;9:44-6.

21. Solish N, Bertucci V, Dansereau A, Hong HC, Lynde C, Lupin M, et al. A comprehensive approach to the recognition, diagnosis, and severity-based treatment of focal hyperhidrosis: Recommendations of the Canadian Hyperhidrosis Advisory Committee. Dermatol Surg. 2007:908:e23.

22. Lear W, Kessler E, Solish N, Glaser DA. An epidemiological study of hyperhidrosis. Dermatol Surg. 2007;33:S69-75.

23. Ou LF, Yan RS, Chen IC, Tang YW.Treatment of axillary bromhidrosis with superficial liposuction. Plast Reconstr Surg. 1998;102:1479- 85.

24. Jemec B, Holm Hansen B. Follow-up of patients operated on for axillary hyperhidrosis by subcutaneous curettage. Scand J Plast Reconstr Surg. 1978;12:65-7.

25. Darabaneanu S, Darabaneanu HA, Niederberger U, Russo PA, Lischner S, Hauschild A. Long-term efficacy of subcutaneous sweat gland suction curettage for axillary hyperhidrosis: A prospective gravimetrically controlled study. Dermatol Surg. 2008;34:1170-7. 